\title{
Weighted Fuzzy Intelligence System in Knowledge Learning System
}

\author{
V. Uma Maheswari ${ }^{1}$, Mr. Jai Kumar ${ }^{2}$ \\ Research Scholar, Department of Computer Science, Sri Ramakrishna Mission Vidyalaya College of Arts and Science, \\ Coimbatore, Tamil Nadu ${ }^{1}$
}

Head of the Department, Department of Computer Applications (UG), Sri Ramakrishna Mission Vidyalaya College of Arts and Science, Coimbatore, Tamil Nadu ${ }^{2}$

\begin{abstract}
Web based education systems are very popular nowadays. Organizing and evaluating user's course details along with their proficiency is very important for improving course systems. With an upgrading technology of ELearning performs a best effect for the student based on the programming language. However, student modeling in many cases deals with uncertainty and it is a difficult process, because the personal skill and ability may vary time to time and person to person. It cannot be exactly said that a learner knows or does not know a particular concept. And also the domain concepts which were previously known by the learner may be completely or partially forgotten. Thus, at present they may be partly known or completely unknown. The teaching process itself changes the status of knowledge of a user. For this problem this paper presents the Weighted Fuzzy intelligence computing for analyzing and helping students about their learning Process and also provides an Adaptive Instruction through E-learning. To accomplish these, the paper introduces a WFIC_KLS (a Weighted Fuzzy Intelligent Computing in Knowledge Learning System), which predicts the relevant course and concepts based on individual proficiency on every concept and suggests best material to improve their skill. The proposed WFIC_KLS helps to find the appropriate tutorial and course link based on individual proficiency. This also effective to guide the learners based on their individual knowledge and interest. The system has successfully implemented using C\#.net platform with a real e learning environment. The experiments and results show the impact of the proposed system in e learning guidance system improves the skill of individual users.
\end{abstract}

Keywords: E-learning system, Data Mining and Survey, Fuzzy Logic, Intelligent Tutoring System, Fuzzy student model, personalization, programming.

\section{INTRODUCTION}

With technical advances in internet over the past decade, the constant use of computer and Internet technologies in the field of education has changed the ways of teaching and learning [1]. This type of enhancements in e-Learning helps everyone to acquire knowledge at any time and from anywhere. Due to this flexible nature, the system also needs a customization in the development, where the user's knowledge state and skill is heterogeneous. So the system should adapt according to the users interest and skill. This fact point outs the need of web-based educational systems development, that can offer dynamic adaptation for every user based on the skill.

Major goal of education is to increase level of intelligence in every individual to progress in all areas. Technological advancements increase the efficiency of decision making and problem solving of human being. To deal with real life problems, certain level of intelligence is essential for every individual. This has been made possible by technological advancements. It has been observed that many times an individual himself cannot identify his own interest and capabilities in specific areas. It has been observed that many times students can- not really identify their capabilities and due to lack of such knowledge; they might select wrong tutorials and samples. In such cases, the users may not get complete advantage of their capabilities. The solution of this problem is to identify the exact capabilities of an individual in the e-learning framework. For optimum utilization of opportunities and to get strategic advantages; it is important to analyze and enhance level of intelligence [2] [3].

In this paper, we bring following points into observation; the major e-learning domains such as w3schools, tutorial sites and tutorial point etc, still lack practical and intelligent decision support system to achieve efficient and powerful classification of human capabilities. Individual student behavior and skill is different, so according to the individual ability, the system needs to perform.

\section{LITERATURE REVIEW}

The Intelligent tutoring system (ITS) is proposed [4] with the Artificial intelligent technique, this gives the knowledge and domain module with student evaluation process, even though the technique is better and providing 
Vol. 5, Issue 12, December 2016

personalization, the authors in the paper doesn't depict how the knowledge of a domain concept may affect by the knowledge of another.

A Personalized recommendation system called as PCRS [5], which is a fuzzy item response theory proposed to determine the course detection difficulty and this is very tough while selecting suitable learning objects on the users.

Finally, IRT (item response theory) is proposed [6]. This paper based on the artificial neural network and it can effectively detect the knowledge state from different dataset and gives an immense accuracy and user friendly platform. However, the system needs several iterations to complete the whole process.

There is a lack of generalized framework implementing Theory of MI (Machine Intelligence) in order to analyze students (i.e. user's in a generalized manner) different skills using evolutionary fuzzy approach. There are many computer based applications developed so far to identify and then enhance different types of intelligence using the Theory of MI. However, the system that can automatically identify specific level of specific type of intelligence using evolving knowledge-base approach through Fuzzy system is yet to be developed.

In order to achieve machine learning and automatic decision making an improved fuzzy approach is developed. This paper is based on supervised machine learning approach in which machine is trained through experts knowledge once, and later on takes decision automatically every time. One of the member of syndicate of SC; Fuzzy Logic is selected to deal with multi-valued classification. A novel personalization system has been designed which integrates linguistic knowledge representation and finally provide the best possible solution automatically. A prime target for such intelligent system is the implementation in the domain of Theory of Multiple Intelligence which analyzes and enhances multiple capabilities in human beings.

\section{PROPOSED SYSTEM}

The paper introduces a WFIC_KLS (a Weighted Fuzzy Intelligent Computing in Knowledge Learning System), which predicts the relevant course and concepts based on individual proficiency on every concept and suggests best material to improve their skill. The proposed WFIC_KLS helps to find the appropriate tutorial and course link based on individual proficiency.

Fuzzy logic has a number of properties that make it suitable for describing e learning events: (i) it can tolerate the unreliable and imprecise datasets; (ii) it is much closer to the way of thinking than crisp logic. (iii) Compared to other classification algorithms based on probability theory, fuzzy logic is much more intuitive and easier to use. A disadvantage of using fuzzy logic is that storing the rule base might require a significant amount of storage. The number of rules grows exponentially to the number of variables. With $n$ variables each of which can take $m$ values, the number of rules in the rule-base is $\mathrm{mn}$. We proposed a new technique to mine e learning data using personalization techniques to extract useful patterns and support to the learners. Fuzzy logic is used with knowledge definer system for effective personalization of rules from quantitative datasets.

\section{Advantages of the proposed system:}

1. The proposed approach improves the efficiency of the adaptively of the instructional process.

2. WFIC_KLS process, which identifies the alterations on the state of students' knowledge level.

3. Student can read the learning material more times, until they have learned the domain knowledge completely.

The goal of this proposal is to personalize the e-learning procedure using rule based fuzzy sets. In order to enhance individual proficiency and adaptive learning process, WFIC_KLS has been proposed.

\section{A. Contributions of the Paper}

Weighted Fuzzy Intelligent Computing in Knowledge Learning System (WFIC_KLS) phase of a web-based educational application is created for individualized instruction on the computer science domains.

The proposed system effectively identifies the individual students skills based on their objective questions, practical results, and the time based evaluations.

The time analysis is one of the factors included to define the student's proficiency in each domain.

WFIC_KLS is the basis for providing personalized tutoring, which identifies and updates the student's knowledge level.

The WFIC_KLS have the ability to recognize the alterations of the student's learning states and dynamically adapt the presentation of the learning material accordingly. In addition the proposed system performs the effective personalized tutoring system. And the detection time and classification time is effectively handled in the proposed system.

\section{B. Weighted Fuzzy Logic:}

Weighted Fuzzy Logic (WFL) is a multi valued paradigm which allows intermediate values to be defined between conventional evaluations like true/false, yes/no, pass/fail, etc. Notions like rather tall or very fast can be formulated mathematically and processed by computers, in order to apply a more human-like way of thinking in the programming of computers. The Fuzzy systems are an alternative to traditional notions of set membership and logic on e learning.

The simplest fuzzy model consists of a set of rules with an "if - then" structure:

If $<$ condition $1>$ and $\ldots$ and $>$ condition $n>$ then $<$ conclusion $>$

Where, condition i is a statement of type " $\mathrm{Zi}$ is Yij". In this statement $\mathrm{Zi}$ represents the actual value of some $i$-th real world variable meanwhile $\mathrm{Yij}$ is a flexible predicate naming the $j$-th linguistic term of the corresponding $i$-th Linguistic Variable. Yij is given by a fuzzy set which 
Vol. 5, Issue 12, December 2016

represents the use of the flexible predicate on the domain of $\mathrm{Zi}$. Statements of this kind are called "premises". The conclusion is also a fuzzy set, which represents the linguistic term expressing a flexible predicate, which characterizes the output behaviour of the system if all conditions are satisfied. Notice that "if - then" rules may be used both to model the state of a system (see Rule 1 below) and to take a decision to control the system (see Rule 2).

Rule 1:If knowledge level (KL) of (level1) is high and the $\mathrm{KL}$ (level2) is high and $\mathrm{KL}$ (level 3) is low then the user need to concentrate on level3.

Rule 2:If knowledge level (KL) of (level1) is medium and $\mathrm{KL}$ (level2) is low. Fuzzy control is then closely related to fuzzy decision making. Take as example the following set of "if - then" rules constituting a fuzzy control-model for an extreme simple e learning system for a particular learner:

R1:If knowledge level (KL) of (level1) is low and the KL (level2) is low and KL (level 3) is low then the user need to concentrate on level1.

R2:If knowledge level (KL) of (level1) is medium and the $\mathrm{KL}$ (level2) is high and KL (level 3) is low then the user need to concentrate on level1.

R3:If knowledge level (KL) of (level1) is high and the KL (level2) is high and KL (level 3) is high then the user need to go on level4.

When a simple database $\mathrm{T}$ with two attributes (X1 and $\mathrm{X} 2$ as level 1 and level 2 respectively) and three linguistic terms and associated MFs (see Figure 1.0) is considered.

\section{Low Medium High $\quad$ Low Medium High}

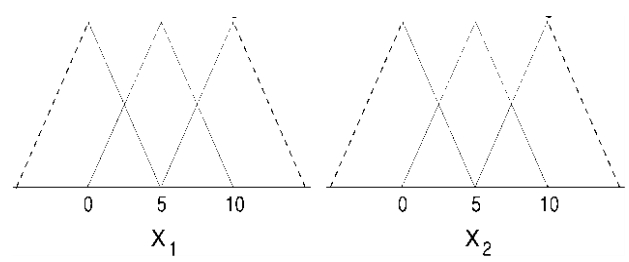

Figure 1.0 Attributes and linguistic terms for the attributes X1 and X2

As per the E-leaning dataset, the above attributes and linguistic terms are defines as the value of individual scores. If the value for $\mathrm{X} 1$ is defined as 5 , then the score will be modified into the linguistic terms (High, medium, Low). This is calculated from the total questions and time taken for every question.

If total question is $10 \rightarrow$ the score is $8 \rightarrow$ the time take for each question is $2 \mathrm{~ms}$ approximately $\rightarrow$ then the linguistic score will be converted as "High".

\section{Domain Knowledge Representation using Fuzzy:}

Domain concepts are the learning material, and directed arcs, which represent relations between the concepts of the learning material. The relations that exist between the concepts of the learning material depict so the order in which the domain concepts have to be delivered and the structure of the learning material, as the knowledge dependencies. In particular, there are three types of relations between the concepts: "precedes" that declares the order in which each domain concept of the learning material has to be taught. A domain concept is affected regarding the knowledge level of its related domain concepts. In other words, they depict the "strength of impact" of a domain concept on a related concept. The particular numbers are only positive [14].

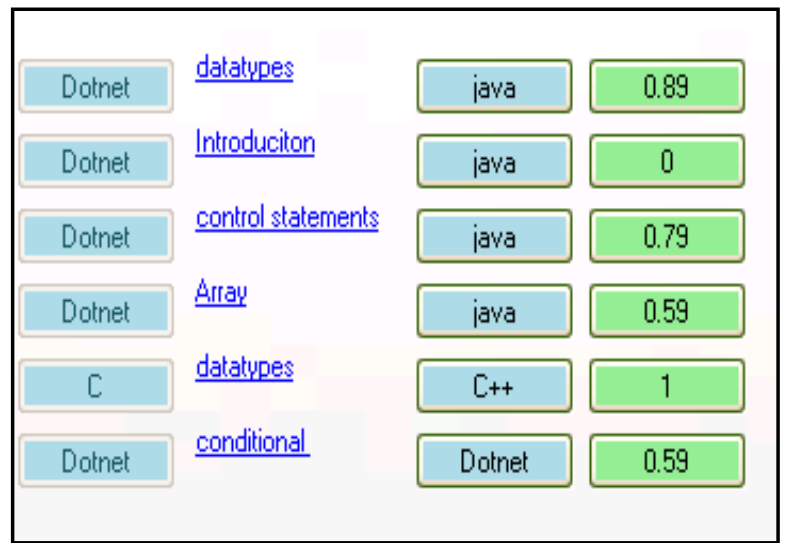

Fig 2.0 concept similarity score calculation in every domain

The Figure 2.0 represents the concept similarities between different programming languages. Using this, the learner can choose the language for further study. This is happened due to the fact that the increase of the knowledge level of a domain concept leads to the increase of the knowledge level of a depended domain concept, and the decrease of the knowledge level of a domain concept leads to the decrease of the knowledge level of a depended domain concept. Therefore, the numbers of the directed arcs that depict the knowledge dependencies belong to the interval $(0,1)$.

One important module of the WFIC_KLS is the domain module, which contains a description of the knowledge or behaviors that represent expertise in the subject-matter domain of the system.

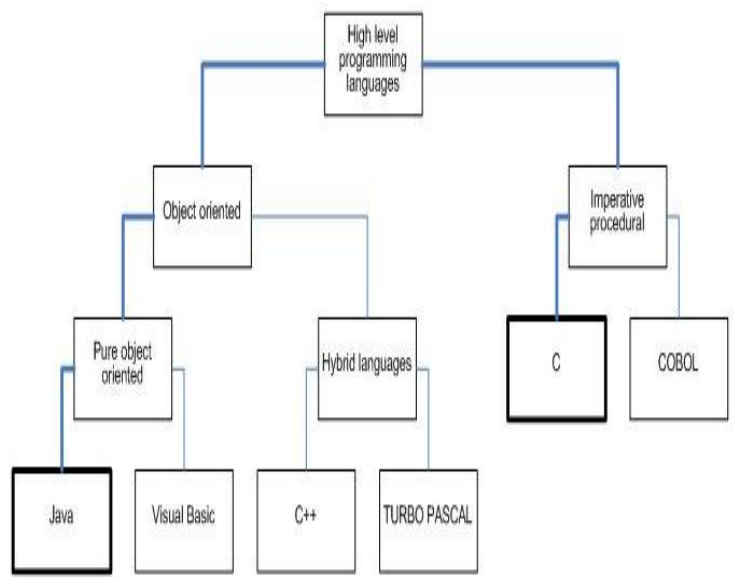

Fig 3.0 Concept Hierarchy 
Vol. 5, Issue 12, December 2016

To enable communication between system and learner at a Knowledge Level: This level includes information about content level, the domain model of the system has to be novice learner current knowledge as opposed to a adequate with respect to inferences and relations of Knowledge sharing systems.

domain entities and compatible.

The hierarchy of the domain concepts, as well as the dependence relations among them must be represented. Therefore, the domain knowledge of the presented system is organized into a hierarchical structure in combination with FCMs.

The proposed algorithm takes less number of iterations to reach a stable state. The time taken by this algorithm is in between the time taken by the existing fuzKSD and time taken by ITS. Dynamic adaptability of the WFIC_KLS model depends on predicting learner's expected results and comparing those results with learner's achieved results.

The results are analyzed to infer the weak points in the plan that needs adaptation. In doing so, the tree of topics included in the plan is traced to calculate the expected learner's results according to his background knowledge mastering levels. The topics are weighted by the instructional designer to determine their relevance importance during the course intake.

\section{Step 2: Domain Knowledge Model}

Knowledge of the domain can be seen as interconnection of two networks which are the network of concepts known as knowledge space and the network of educational material. Knowledge space is a structured representation of the domain knowledge, in our proposal the score has been used for knowledge representation. It consists of interconnected nodes where each node represents a small piece of knowledge (i.e. a concept).

\section{Course Model}

The adaptation capability of the proposed system highly depends on the past successful course plans stored in the database along with the scores. The course model is a structured case-based model, where each case represents an experience of course delivery.

Every learner can view their domain knowledge on each domain. Finally the proposed system will provide appropriate tutorials and notes to improve their knowledge.

\section{Step 3: Evaluation model}

\section{Steps:}

Step1: Learner Level

Researchers in the e-learning community have always considered it important to develop a model of the novice learner. The adaptive and collaborative capabilities of the system are mainly based on this model. Along with many characteristics that define the learner model there are characteristics that have a great influence on the learning process such are:

1. Prior knowledge of the learner that determines the concepts that he can learn.

2. Learner's skills and learning styles that determines the teaching approach that best suits the learner.

3. Learner's emotions and motivations is another important characteristic that is usually forgotten in learner modelling research.

Thus, we proposed a multi-level comprehensive learner model that is based on the Novice learner Information Package (NIP). The proposed novice learner model consists of three levels as follows.

Personality level: This level consists of information related to the learner type and any extensions to it, along with information about the learning style and skills of the novice learner.

Behaviour Level: This level is responsible for recording information about learner activities in the system. For example test and time taken for each test has been analyzed.

Each training state was designed with specific issues in mind. The evaluation of past training cases includes calculating the similarity measures between the state of current learner and the previous training cases. This process should consider all the issues that affect the construction of the previous training state. These issues include the training objectives, learner's knowledge mastering levels, his skills appraisal levels, his learning style along with any possible effective environmental issues. Moreover, looking at past learners' achieved results may be helpful in choosing the best previous training experience.

It is worth noting that each of the above issues has elearning own relative impact on the training experience. The relative importance of each issue can be set by the system administrator.

The problem is how to evaluate previous training cases in order to choose the training state that has the highest degree of similarity to novice learner 1 . First, let us suppose that $E=\left\{e_{1}, e_{2}, \ldots, e_{n}\right\}$ is a set of n-effective issues. Second, assume that $\mathrm{L}=\left\{1_{1}, l_{2}, \ldots, \mathrm{l}_{\mathrm{k}}\right\}$ is a set of $\mathrm{k}$ previous learning cases, while 1 by e-learning elf will be used to indicate the current learning state. Moreover, the similarity between the effect of $e_{i}$ on the current learning state and e-learning effect on previous learning state $l_{p}$ is $\mathrm{S}_{\mathrm{i}}\left(1, \mathrm{l}_{\mathrm{p}}\right) . \mathrm{R}=\left\{\mathrm{r}_{1}, \mathrm{r}_{2}, \ldots, \mathrm{r}_{\mathrm{n}}\right\}$ is a set of relative importance values corresponding to different effective issues in $\mathrm{E}$; so that $r_{j}$ is the relative importance of issue $e_{j}$; where $\left(r_{j}<1\right)$ and this will be applied in every dataset for fast skill evaluation. Finally the weighted fuzzy gives the learning state with reliable tutor detection. 


\section{RESULTS AND DISCUSSION}

The WFIC_KLS is successfully implemented in C\#.net with real-time student's dataset, which have collected from numerous students. The students learning details are collected from the created website. The website crawled students studying behaviors, exams attended and marks obtained with time taken for each question and domain. From the details the student knowledge state is analyzed. The table 1.0 shows, the impact of the proposed system in two domain level. This has been calculated for Learner A.

Table 1.0 learners count in different stereo type

\begin{tabular}{|c|c|c|}
\hline Type & FuzKSD(existing) & WFIC_KLS(proposed) \\
\hline S3.1 & 60 & 62 \\
\hline S3.2 & 49 & 51 \\
\hline S3.3 & 27 & 29 \\
\hline S3.4 & 37 & 37 \\
\hline S3.5 & 20 & 20 \\
\hline S4.1 & 60 & 65 \\
\hline S4.2 & 27 & 28 \\
\hline S4.3 & 23 & 24 \\
\hline S4.4 & 28 & 28 \\
\hline S4.5 & 49 & 51 \\
\hline
\end{tabular}

The table 1.0 shows the stereo type and score before stereo type 3 and after stereo type 5 .

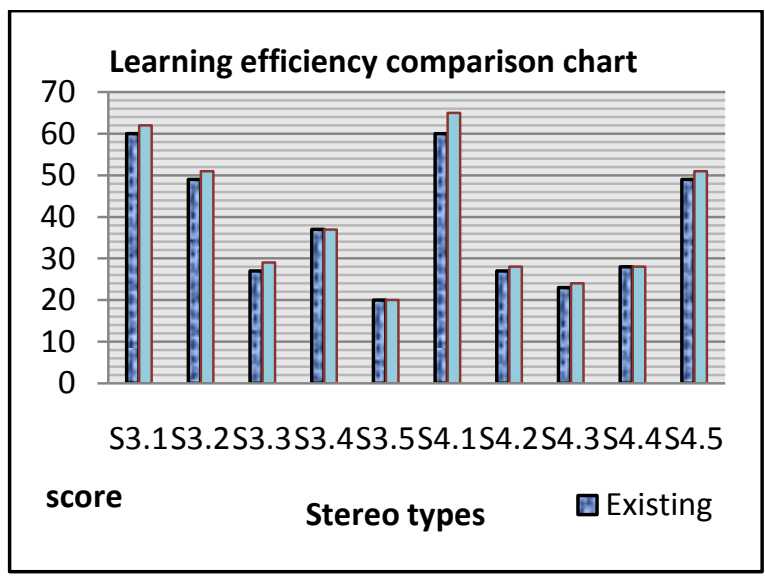

Fig 4.0 changes in knowledge level in WFIC_KLS

The chart 4.0 shows, the stereo type 3 score and stereo type 5 score before and after implementing the proposed system. The score is increased after implementing WFIC_KLS. That is best than the existing system.

\section{Rule Generation comparison:}

The rule generation comparison process is performed to analyze the efficiency of the proposed system with the existing system in terms of time. This comparison made between existing fuzzy logic process and proposed weighted fuzzy logic process.

The chart 5.0 represents the comparison chart for the rule generation time between existing and proposed system

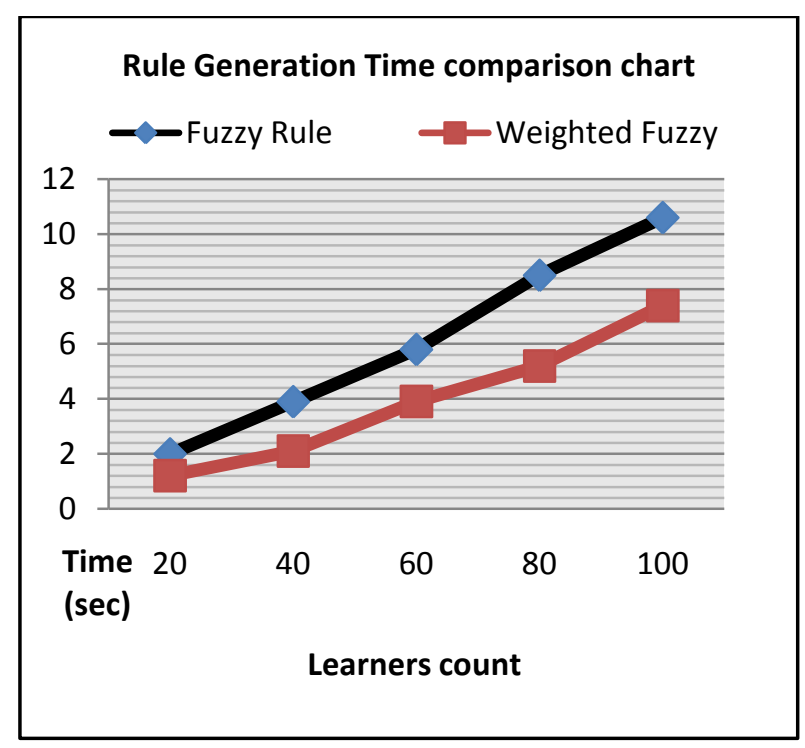

Fig 5.0 Rule generation time comparison chart

\section{CONCLUSION}

E-Learning is an emerging field which provides education through web through programming language. All learners should not be forced to read the same material or in the same order. Some learners do not need to read every domain concept because they are known to them while others have to be revised. Finally, the teaching process itself changes the status of knowledge of a learner. This happens due to the fact that a learner accepts new concepts while being taught. For this issue this paper shows the Weighted Fuzzy intelligence computing for automatically learning Process for the Student to provide an Adaptive Instruction through E-learning.

The goal of this proposed system is to show how fuzzy sets can be combined with learner stereotypes and the different model to promote adaptively and personalization in educational applications with different decision schemes.

For the purpose of this research, a novel educational system was fully implemented and evaluated, regarding the innovative approach described. More specifically, the paper presents the development and use of the WFIC_KLS module, which identifies the alterations on the state of students' knowledge level. WFIC_KLS operates in collaboration with FCMs that are used to represent the dependences among the domain concepts. WFIC_KLS monitors the learner's performance in a domain concept, updates the learner's overlay model, and makes dynamic decisions on how the teaching syllabus is presented to the learner to fit his/her personal needs. In particular, when a change occurs on a learner's knowledge level of a domain concept, WFIC_KLS identifies and updates the student's knowledge level not only for this concept, but also for all the related concepts with this concept, considering the learner's performance, as well as the existing dependences among the domain concepts in the FCMs. 


\section{REFERENCES}

[1] J. Lo, Y. Chan, and S. Yen, "Designing an adaptive web-based learning system based on students' cognitive styles identified online," ComputEdu., vol. 58, no. 1, pp. 209-222, Jan. 2012.

[2] Paramythis, Alexandros, and Susanne Loidl-Reisinger. "Adaptive learning environments and e-learning standards." Second european conference on e-learning. Vol. 1. 2003.

[3] Sterbini, Andrea, and Marco Temperini. "Adaptive construction and delivery of web-based learning paths." Frontiers in Education Conference, 2009. FIE'09. 39th IEEE. IEEE, 2009.

[4] SalisuSani et al, "Proposal for Ontology Based Approach to Fuzzy Student Model Design".

[5] Chen, Chih-Ming, Ling-Jiun Duh, and Chao-Yu Liu. "A personalized courseware recommendation system based on fuzzy item response theory."e-Technology, e-Commerce and e-Service, 2004. EEE'04. 2004 IEEE International Conference on. IEEE, 2004.

[6] Baylari, Ahmad, and Gh A. Montazer. "Design a personalized elearning system based on item response theory and artificial neural network approach." Expert Systems with Applications 36.4 (2009): 8013-8021.

\section{BIOGRAPHIES}

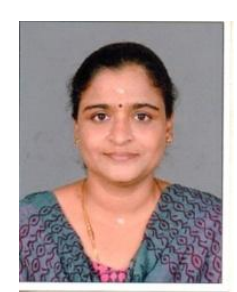

Mrs. V. Uma Maheswari completed MCA and currently pursuing M. Phil in Computer Science at Sri Ramakrishna Mission Vidyalaya College of Arts and Science, Coimbatore-20. She worked as a Teacher In Indian School -Tanzaia, East Africa. Her Teaching Experience mining Web mining. is 5 years. Her area of interest is Data

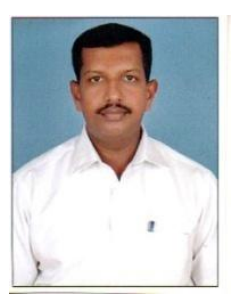

M. Jai Kumar completed MCA at Bharathiar University, M. Phil at Bharathiar University and currently pursuing Ph. D in Sri Ramakrishna Mission Vidyalaya College of Arts and Science, Coimbatore-20. He is working as Assistant Professor\& Head of the Dept. of Computer Applications Department (UG) in Sri Ramakrishna Mission Vidyalaya College of Arts and Science, Coimbatore. His teaching experience is 11 years. His area of interest is Software Engineering and Data Mining 